\title{
THE $\ell^{2}$-COHOMOLOGY OF ARTIN GROUPS
}

\author{
M. W. DAVIS AND I. J. LEARY
}

\begin{abstract}
For each Artin group we compute the reduced $\ell^{2}$ cohomology of (the universal cover of) its "Salvetti complex". This is a CW-complex which is conjectured to be a model for the classifying space of the Artin group. In the many cases when this conjecture is known to hold our calculation describes the reduced $\ell^{2}$-cohomology of the Artin group.
\end{abstract}

\section{INTRODUCTION}

Suppose that $G$ is a countable discrete group acting properly and cellularly on a CW-complex $E$ with compact quotient. As in [14] or [16] one can define the reduced $\ell^{2}$-cohomology group $\mathcal{H}^{i}(E)$ for each non-negative integer $i$. (We will give the definition in Section 3.) For each $i, \mathcal{H}^{i}(E)$ is a Hilbert space with an orthogonal $G$-action. When $G$ is infinite, these Hilbert spaces tend to be infinite-dimensional unless they are zero. However, each $\mathcal{H}^{i}(E)$ is a "Hilbert $G$-module", and to any Hilbert $G$-module $V$ one may associate a real number, called its von Neumann dimension and denoted by $\operatorname{dim}_{G}(V)$. One can then define the $\ell^{2}$-Betti numbers of $E$ with respect to $G$ by

$$
\ell^{2} b_{i}(E ; G):=\operatorname{dim}_{G} \mathcal{H}^{i}(E) .
$$

If $E$ is contractible and $G$ acts freely on $E$ (so that $E / G$ is a classifying space for $G$ ), these $\ell^{2}$-cohomology groups (and their von Neumann dimensions) are independent of the choice of $E$ and so are invariants of $G$. In this case we will use the notation $\mathcal{H}^{i}(G)$ and $\ell^{2} b_{i}(G)$ to stand for $\mathcal{H}^{i}(E)$ and $\ell^{2} b_{i}(E ; G)$ respectively.

$\ell^{2}$-cohomology groups have proved difficult to compute directly. To date, most of the calculations have been based on two principles: (i) certain vanishing theorems; (ii) Atiyah's formula. A typical vanishing

Date: October 13, 2011.

2000 Mathematics Subject Classification. Primary: 57M07; Secondary: 20F36, 20J05.

The first author was partially supported by NSF grant DMS-0104026. The second author acknowledges support from EPSRC (via grant GR/R07813), and from the Ohio State Mathematical Research Institute. 
theorem will assert that for a certain class of groups $G, \mathcal{H}^{i}(G)$ is zero for $i$ within a certain range. Atiyah's formula states that in the case when $G$ acts freely on $E$, the alternating sum of the $\ell^{2}$-Betti numbers of $G$ is equal to the ordinary Euler characteristic of $E / G$. In cases where $\mathcal{H}^{*}(E)$ is known to vanish except in one dimension, this formula provides an exact calculation of the unique non-zero $\ell^{2}$-Betti number. In this paper we compute the $\ell^{2}$-Betti numbers $\ell^{2} b_{i}(G)$ when $G$ is an Artin group. Surprisingly, there is a completely clean answer which is non-trivial in the sense that many of the $\ell^{2} b_{i}(G)$ are non-zero.

Associated to any Coxeter matrix $\left(m_{i j}\right)$ on a finite set $I$, there is a Coxeter group $W$, an Artin group $A$ and a simplicial complex $L$ with vertex set $I$, called the "nerve" of the Coxeter matrix. (The definitions will be given in Section 5.) Coxeter groups are intimately connected to groups generated by reflections. For example, if $n=\operatorname{Card}(I)$, there is a standard representation of $W$ as a linear reflection group on $\mathbb{R}^{n}$, so that there is a certain convex cone in $\mathbb{R}^{n}$ with $W$ acting properly on its interior $\Omega$.

Artin groups have a related geometric interpretation. Consider the complexification of the $W$-representation described above. Then $\mathbb{R}^{n}+$ $i \Omega$ is a a $W$-stable open convex subset of $\mathbb{C}^{n}$ on which $W$ acts properly. Moreover, $W$ acts freely on the hyperplane complement

$$
Y=\left(\mathbb{R}^{n}+i \Omega\right)-\bigcup_{r} H_{r}
$$

where $r$ ranges over all reflections in $W$ and $H_{r}$ denotes the intersection of the complex hyperplane fixed by $r$ and $\left(\mathbb{R}^{n}+i \Omega\right)$. It is proved in [7] that there is a finite CW-complex $X$, called the "Salvetti complex" which is homotopy equivalent to $Y / W$. The complex $X$ has one vertex and, for each $k>0$, one $k$-cell for each $(k-1)$-simplex of $L$. The 2-skeleton of $X$ is the presentation complex associated to the standard presentation of $A$. It follows that $\pi_{1}(Y / W)=\pi_{1}(X)=A$. It is conjectured that the universal cover $\widetilde{X}$ of $X$ is contractible, or equivalently that $X$ is a classifying space $B A$ for $A$. Deligne [12] proved this conjecture in the case when $W$ is finite. The conjecture is also known to be true when $W$ is "right-angled", when $\operatorname{dim} L \leq 1$, and in many other cases (see $[6,7])$. Our actual calculation is of $\mathcal{H}^{i}(\widetilde{X})$. So our title is somewhat misleading: we only have a computation of the reduced $\ell^{2}$-cohomology of $A$ modulo the conjecture that $X=B A$.

Theorem 1. For any Artin group $A$ and any $i$, there is an isomorphism of Hilbert A-modules:

$$
\mathcal{H}^{i}(\widetilde{X}) \cong \bar{H}^{i-1}(L) \otimes \ell^{2}(A) .
$$


Corollary 2. $\ell^{2} b_{i}(\widetilde{X} ; A)=\bar{b}_{i-1}(L)$.

Here $\bar{b}_{j}(L)$ denotes the ordinary "reduced Betti number" of $L$, i.e., $\bar{b}_{j}(L)$ is the dimension of the reduced homology group $\bar{H}_{j}(L ; \mathbb{R})$.

When $W$ is finite, the Artin group $A$ is said to be "spherical". If $A$ is spherical, then its centre always has an infinite cyclic subgroup (coming from the $\mathbb{C}^{*}$-action on the hyperplane complement). For such groups the reduced $\ell^{2}$-cohomology is known to vanish (see Section 4). Hence, in the spherical case, Theorem 1 is well-known. This vanishing result in the spherical case is the key ingredient for our proof in the general case.

In fact, a stronger result that Theorem 1 is true. In the following statement, the "standard homomorphism" from an Artin group to $\mathbb{Z}$ is the homomorphism that sends each Artin generator to $1 \in \mathbb{Z}$.

Theorem 3. Suppose that $A^{\prime}$ is a normal subgroup of the Artin group $A$ that is contained in the kernel of the standard homomorphism $A \rightarrow \mathbb{Z}$. Let $X^{\prime} \rightarrow X$ be the corresponding covering space of $X$ and let $G=$ $A / A^{\prime}$. Then $\mathcal{H}^{i}\left(X^{\prime}\right)$ and $\bar{H}^{i-1}(L) \otimes \ell^{2}(G)$ are isomorphic as Hilbert $G$-modules. In particular, $\ell^{2} b_{i}\left(X^{\prime} ; G\right)=\bar{b}_{i-1}(L)$.

Theorem 3 was suggested by a result of [15].

\section{Hilbert $G$-Modules}

Let $G$ be a countable discrete group. The Hilbert space of squaresummable real-valued functions on $G$ is denoted by $\ell^{2}(G)$. There is an orthogonal (right) action of $G$ on $\ell^{2}(G)$, and a diagonal action on any direct sum of copies of $\ell^{2}(G)$. A Hilbert space $V$ equipped with an orthogonal(right) $G$-action is called a (finitely generated) Hilbert $G$ module if it is $G$-equivariantly isomorphic to a $G$-stable closed subspace of the direct sum of finitely many copies of $\ell^{2}(G)$. A map $f: V \rightarrow V^{\prime}$ of Hilbert $G$-modules is by definition a $G$-equivariant bounded linear operator. The kernel of $f: V \rightarrow V^{\prime}$, denoted $\operatorname{Ker}(f)$, is a closed subspace of $V$, but the image, $\operatorname{Im}(f)$, need not be closed. The map $f$ is weakly surjective if $\overline{\operatorname{Im}(f)}=V$; $f$ is a weak isomorphism if it is both injective and weakly surjective.

To each Hilbert $G$-module $V$ it is possible to associate a non-negative real number $\operatorname{dim}_{G}(V)$, called its von Neumann dimension. The three most important properties of $\operatorname{dim}_{G}(-)$ are:

(1) $\operatorname{dim}_{G}(V)=0$ if and only if $V=0$;

(2) $\operatorname{dim}_{G}\left(V \oplus V^{\prime}\right)=\operatorname{dim}_{G}(V)+\operatorname{dim}_{G}\left(V^{\prime}\right)$;

(3) $\operatorname{dim}_{G}\left(\ell^{2}(G)\right)=1$. 
It follows from (1) that for any map $f: V \rightarrow V^{\prime}$,

$$
\operatorname{dim}_{G}(V)=\operatorname{dim}_{G}(\operatorname{Ker}(f))+\operatorname{dim}_{G}(\overline{\operatorname{Im}(f)}) .
$$

Using property (1), one can then deduce the following.

Lemma 4. Suppose that $V$ and $V^{\prime}$ are Hilbert $G$-modules and that $\operatorname{dim}_{G}(V)=\operatorname{dim}_{G}\left(V^{\prime}\right)$. The following are equivalent:

(a) $f$ is injective;

(b) $f$ is weakly surjective;

(c) $f$ is a weak isomorphism.

Proof. By definition, (c) is equivalent to the conjunction of (a) and (b). Thus it suffices to show that (a) and (b) are equivalent. But we have that $\operatorname{dim}_{G}(\overline{\operatorname{Im}(f)})=\operatorname{dim}_{G}(V)-\operatorname{dim}_{G}(\operatorname{Ker}(f))$, and so $\operatorname{dim}_{G}(\operatorname{Ker}(f))=$ 0 if and only if $\operatorname{dim}_{G}(\overline{\operatorname{Im}(f)})=\operatorname{dim}_{G}(V)=\operatorname{dim}_{G}\left(V^{\prime}\right)$.

Next suppose that $C^{*}=\left\{\left(C^{n}, \delta_{n}\right)\right\}$ is a cochain complex of Hilbert $G$-modules, i.e., the coboundary maps $\delta_{i}: C^{i} \rightarrow C^{i+1}$ are $G$-equivariant bounded linear operators. The cohomology group

$$
H^{i}\left(C^{*}\right):=\operatorname{Ker}\left(\delta_{i}\right) / \operatorname{Im}\left(\delta_{i-1}\right)
$$

need not be a Hilbert space since $\operatorname{Im}\left(\delta_{i-1}\right)$ is not necessarily closed in $\operatorname{Ker}\left(\delta_{i}\right)$. However, the reduced cohomology groups defined as

$$
\mathcal{H}^{i}\left(C^{*}\right):=\operatorname{Ker}\left(\delta_{i}\right) / \overline{\operatorname{Im}\left(\delta_{i-1}\right)}
$$

are Hilbert $G$-modules.

Lemma 5. Suppose that $\left(C^{*}, \delta_{*}\right)$ and $\left(C^{\prime *}, \delta_{*}^{\prime}\right)$ are cochain complexes of Hilbert $G$-modules and that $f: C^{*} \rightarrow C^{\prime *}$ is a weak isomorphism of cochain complexes (i.e., $f$ is a cochain map and for each $i, f_{i}: C^{i} \rightarrow C^{\prime i}$ is a weak isomorphism). Then the induced map $\mathcal{H}(f): \mathcal{H}^{i}\left(C^{*}\right) \rightarrow$ $\mathcal{H}^{i}\left(C^{\prime *}\right)$ is also a weak isomorphism. In particular, $\mathcal{H}^{i}\left(C^{*}\right)$ and $\mathcal{H}^{i}\left(C^{*}\right)$ are isometric Hilbert $G$-modules.

Proof. Let $Z^{i}$ and $B^{i}$ (resp. $Z^{\prime i}$ and $B^{\prime i}$ ) denote the cocycles and coboundaries in $C^{*}$ (resp. $\left.C^{\prime *}\right)$. Since $f$ is a cochain map, $f\left(Z^{i}\right) \subseteq Z^{\prime i}$ and $f\left(B^{i}\right) \subseteq B^{\prime i}$. Moreover, since $f$ is continuous, $f\left(\overline{B^{i}}\right) \subseteq \overline{{B^{\prime}}^{i}}$. Since $f$ is injective this gives

$$
\operatorname{dim}_{G}\left(Z^{\prime i}\right) \geq \operatorname{dim}_{G}\left(Z^{i}\right) \quad \text { and } \operatorname{dim}_{G}\left({B^{\prime}}^{i}\right) \geq \operatorname{dim}_{G}\left(B^{i}\right) .
$$

From the short exact sequences

$$
\begin{gathered}
0 \rightarrow Z^{i} \rightarrow C^{i} \rightarrow B^{i+1} \rightarrow 0 \\
0 \rightarrow Z^{\prime i} \rightarrow C^{\prime i} \rightarrow B^{\prime i+1} \rightarrow 0
\end{gathered}
$$


one obtains

$$
\begin{gathered}
\operatorname{dim}_{G}\left(C^{i}\right)=\operatorname{dim}_{G}\left(Z^{i}\right)+\operatorname{dim}_{G}\left(B^{i+1}\right), \quad \text { and } \\
\operatorname{dim}_{G}\left(C^{\prime i}\right)=\operatorname{dim}_{G}\left(Z^{\prime i}\right)+\operatorname{dim}_{G}\left(B^{\prime i+1}\right)
\end{gathered}
$$

Since $f$ is a weak isomorphism, one has that $\operatorname{dim}_{G}\left(C^{\prime i}\right)=\operatorname{dim}_{G}\left(C^{i}\right)$. Hence

$$
\begin{aligned}
\operatorname{dim}_{G}\left(C^{i}\right) & =\operatorname{dim}_{G}\left(C^{\prime i}\right)=\operatorname{dim}_{G}\left(Z^{\prime i}\right)+\operatorname{dim}_{G}\left(B^{\prime i+1}\right) \\
& \geq \operatorname{dim}_{G}\left(Z^{i}\right)+\operatorname{dim}_{G}\left(B^{i+1}\right)=\operatorname{dim}_{G}\left(C^{i}\right)
\end{aligned}
$$

which together with (1) implies that

$$
\operatorname{dim}_{G}\left(Z^{\prime i}\right)=\operatorname{dim}_{G}\left(Z^{i}\right) \text { and } \operatorname{dim}_{G}\left(B^{\prime i}\right)=\operatorname{dim}_{G}\left(B^{i}\right) .
$$

It follows from Lemma 4 that the maps $f: Z^{i} \rightarrow Z^{\prime i}$ and $f: B^{i} \rightarrow B^{\prime i}$ are weak isomorphisms. This implies that $\mathcal{H}(f): \mathcal{H}^{i}\left(C^{*}\right) \rightarrow \mathcal{H}^{i}\left(C^{\prime *}\right)$ is weakly surjective.

To show that $\mathcal{H}(f)$ is injective, let $H$ be the orthogonal complement to $\overline{B^{i}}$ in $Z^{i}$. Note that $H$ is closed and $G$-stable. Now

$$
\begin{aligned}
\operatorname{dim}_{G}\left(Z^{\prime i}\right) & =\operatorname{dim}_{G}\left(\overline{f\left(H+\overline{B^{i}}\right)}\right) \\
& =\operatorname{dim}_{G}(\overline{f(H)})+\operatorname{dim}_{G}\left(\overline{{B^{\prime}}^{\prime}}\right)-\operatorname{dim}_{G}\left(\overline{f(H)} \cap \overline{{B^{\prime}}^{i}}\right) \\
& =\operatorname{dim}_{G}\left(Z^{\prime i}\right)-\operatorname{dim}_{G}\left(\overline{f(H)} \cap \overline{B^{\prime \prime}}\right),
\end{aligned}
$$

where the third equality follows from (3). Hence $\operatorname{dim}_{G}\left(\overline{f(H)} \cap \overline{{B^{\prime}}^{i}}\right)=$ 0 , and therefore $\overline{f(H)}$ is a complementary subspace for $\overline{B^{\prime \prime}}$. Since $f$ is injective when restricted to $H$, it follows that so is $\mathcal{H}(f)$. (This follows from the fact that any two weakly isomorphic Hilbert $G$-modules are in fact equivariantly isometric [14].)

\section{Cohomology with local Coefficients And $\ell^{2}$-COHOMOLOGY}

Suppose that $Y$ is a connected $\mathrm{CW}$-complex with fundamental group $\pi$ and universal cover $\widetilde{Y}$. Suppose also that $Y$ is of finite type (i.e., has finite skeleta.) Let $C_{*}(\widetilde{Y})$ denote the chain complex of $\widetilde{Y}$, which is a chain complex of finitely generated free (left) $\mathbb{Z} \pi$-modules. By a local coefficient system on $Y$ we shall mean a (left) $\mathbb{Z} \pi$-module. The cochain complex for $Y$ with coefficients $M$ is defined by

$$
C^{*}(Y ; M):=\operatorname{Hom}_{\mathbb{Z} \pi}\left(C_{*}(\tilde{Y}), M\right) .
$$


Let $Y^{\prime} \rightarrow Y$ be a regular covering space corresponding to a normal subgroup $\pi^{\prime}$ of $\pi$, and let $G$ denote the quotient group $\pi / \pi^{\prime}$. Then $\ell^{2}(G)$ may be viewed as a $\pi$-G-bimodule, where the left action of $\pi$ is via the action of $G$. The $\ell^{2}$-cochains on $Y^{\prime}$ are defined by

$$
\begin{aligned}
\ell^{2} C^{*}\left(Y^{\prime}\right) & :=\operatorname{Hom}_{\mathbb{Z} G}\left(C_{*}\left(Y^{\prime}\right), \ell^{2}(G)\right) \\
& =\operatorname{Hom}_{\mathbb{Z} \pi}\left(C_{*}(\widetilde{Y}), \ell^{2}(G)\right) \\
& =C^{*}\left(Y ; \ell^{2}(G)\right) .
\end{aligned}
$$

Note that the orthogonal right action of $G$ on $\ell^{2}(G)$ makes $\ell^{2} C^{*}\left(Y^{\prime}\right)$ a chain complex of Hilbert $G$-modules. The unreduced and reduced cohomology groups of $\ell^{2} C^{*}\left(Y^{\prime}\right)$ will be denoted $\ell^{2} H^{*}\left(Y^{\prime}\right)$ and $\mathcal{H}^{*}\left(Y^{\prime}\right)$ respectively. The $\ell^{2}$-Betti numbers of $Y^{\prime}$ with respect to $G$ are then defined by $\ell^{2} b_{i}\left(Y^{\prime} ; G\right):=\operatorname{dim}_{G} \mathcal{H}^{i}\left(Y^{\prime}\right)$.

In the case when $Y^{\prime}$ is acyclic, $C_{*}\left(Y^{\prime}\right)$ is a free resolution for $\mathbb{Z}$ over $\mathbb{Z} G$. Any two such resolutions are chain homotopy equivalent. Similarly, if $Y^{\prime}$ is only rationally acyclic, the rational chain complex $C_{*}\left(Y^{\prime}, \mathbb{Q}\right)$ is a free resolution for $\mathbb{Q}$ over $\mathbb{Q} G$, and is unique up to chain homotopy equivalence. It follows that in the case when $Y^{\prime}$ is rationally acyclic, the $\ell^{2}$-cohomology groups and $\ell^{2}$-Betti numbers depend only on $G$ (and not on the particular choice of $Y^{\prime}$ ). In this case we shall use the notation

$$
\begin{aligned}
\ell^{2} H^{i}(G) & :=\ell^{2} H^{i}\left(Y^{\prime}\right), \\
\mathcal{H}^{i}(G) & :=\mathcal{H}^{i}\left(Y^{\prime}\right), \\
\ell^{2} b_{i}(G) & :=\operatorname{dim}_{G} \mathcal{H}^{i}\left(Y^{\prime}\right) .
\end{aligned}
$$

(Of course, we have in mind the case when $G=\pi$ and $Y^{\prime}=\tilde{Y}$ is contractible.)

\section{A VANishing THEOREM}

Let $G$ be a group and $Z$ a central subgroup of $G$. Any (left) $G$ module $M$ may be viewed as a $G$ - $Z$-bimodule where the right action of $z \in Z$ is defined by $m z=z m$. This induces a right action of $Z$ on $H^{*}(G ; M)$. However one has:

Proposition 6. With notation as above, the action of $Z$ on $H^{*}(G ; M)$ is trivial.

Proof. Let $P_{*}$ be a free resolution for $\mathbb{Z}$ over $\mathbb{Z} G$, so that $H^{*}(G ; M)$ may be computed using the cochain complex $\operatorname{Hom}_{G}\left(P_{*}, M\right)$. In terms 
of this cochain complex the right action of $z \in Z$ on $H^{*}(G ; M)$ sends $f \in \operatorname{Hom}_{G}\left(P_{i}, M\right)$ to $z f$, defined by $z f(p)=z(f(p))$ for all $p \in P_{i}$. It suffices to show that this map is chain-homotopic to the identity map. Since $z$ is central, the map $P_{*} \rightarrow P_{*}$ given by $p \mapsto z p$ is a chain map, and this chain map lifts the identity map from $\mathbb{Z}$ to $\mathbb{Z}$, so is chainhomotopic to the identity map of $P_{*}$. But for all $f \in \operatorname{Hom}_{G}\left(P_{*}, M\right)$ and all $p \in P_{*}, z f(p)=f(z p)$, and so the two actions of $Z$ (on $M$ and on $P_{*}$ ) induce the same action on $\operatorname{Hom}_{G}\left(P_{*}, M\right)$.

Corollary 7. Let $\pi$ be a group admitting a finite classifying space $Y$, let $\pi^{\prime}$ be a normal subgroup of $\pi$, let $G=\pi / \pi^{\prime}$, and suppose that the centre of $\pi$ contains an element whose image in $G$ has infinite order. Then $\mathcal{H}^{*}\left(Y^{\prime}\right)=\{0\}$, where $Y^{\prime}$ denotes the regular cover of $Y$ corresponding $\pi^{\prime}$.

Proof. Let $Z$ denote an infinite cyclic subgroup of the centre of $\pi$ with $Z \cap \pi^{\prime}=\{1\}$. The usual right action of $Z$ on $\ell^{2}(G)$ and the action used in Proposition 6 agree, and hence the right action of $Z$ on $\ell^{2} H^{*}\left(Y^{\prime}\right)$ is trivial. It follows that the action of $Z$ on $\mathcal{H}^{*}\left(Y^{\prime}\right)$ is trivial. Since the image of $Z$ in $G$ is infinite, no non-zero element of $\ell^{2}(G)$ is fixed by the right action of $Z$. However, each $\mathcal{H}^{i}\left(Y^{\prime}\right)$ is a Hilbert $G$-module, and so one obtains a contradiction unless each $\mathcal{H}^{i}\left(Y^{\prime}\right)=\{0\}$.

Corollary 8. Suppose that $G$ has a finite classifying space and that the centre of $G$ contains an element of infinite order. Then the $\ell^{2}$ cohomology of $G$ vanishes.

Proof. This is just the case $G=\pi$ of Corollary 7 .

Other more general vanishing theorems for $\ell^{2}$-cohomology may be found in $[8,16,19,20]$.

\section{Coxeter groups And Artin groups}

Let $I$ be a finite set. A Coxeter matrix $M=\left(m_{i j}\right)$ on $I$ is an $I$-by- $I$ symmetric matrix with entries in $\mathbb{N} \cup\{\infty\}$ such that each $m_{i i}=1$ and such that whenever $i \neq j, m_{i j} \geq 2$. Associated to $M$ there is a Coxeter group denoted by $W_{I}$ or $W$ with generating set $\left\{s_{i}: i \in I\right\}$, with relations

$$
\left(s_{i} s_{j}\right)^{m_{i j}}=1 \quad \text { for all }(i, j) \in I \times I .
$$

There is also an Artin group $A_{I}$ (or $A$ ) with generators $\left\{a_{i}: i \in I\right\}$ and with a presentation given by the relations

$$
a_{i} a_{j} \cdots=a_{j} a_{i} \cdots
$$


for all $i \neq j$, where there are $m_{i j}$ terms on each side of the equation. In both the Coxeter and Artin cases, the relation is interpreted as being vacuous if $m_{i j}=\infty$.

Since each of the Artin relators contains the same number of copies of a generator on each side, there is a homomorphism from any Artin group to the infinite cyclic group $\mathbb{Z}$, defined by sending each Artin generator to 1 . Call this the standard homomorphism from $A$ to $\mathbb{Z}$.

Let $q: A \rightarrow W$ denote the natural homomorphism sending $a_{i}$ to $s_{i}$. There is a set-theoretic section for $q$ denoted $w \mapsto a_{w}$ which may be defined as follows: if $s_{i_{1}} \cdots s_{i_{n}}$ is any word of minimal length for $w$ in terms of the $s_{i}$, then $a_{w}:=a_{i_{1}} \cdots a_{i_{n}}$. As explained in [6, p. 602], it follows from Tits' solution to the word problem for Coxeter groups that $w \mapsto a_{w}$ is well-defined.

Given a subset $J$ of $I$, let $M_{J}$ denote the minor of $M$ whose rows and columns are indexed by $J$, and let $W_{J}$ (resp. $A_{J}$ ) be the corresponding Coxeter group (resp. Artin group). It is known (cf. [2]) that the natural map $W_{J} \rightarrow W_{I}$ (resp. $\left.A_{J} \rightarrow A_{I}\right)$ is injective, and hence $W_{J}\left(\operatorname{resp} . A_{J}\right.$ ) can be identified with the subgroup of $W_{I}$ (resp. $A_{I}$ ) generated by $\left\{s_{i}: i \in J\right\}$ (resp. $\left\{a_{i}: i \in J\right\}$ ).

Say that the subset $J \subseteq I$ is spherical if $W_{J}$ is finite. If this is the case, then the groups $W_{J}$ and $A_{J}$ are both called spherical. (Note that this differs from the terminology of [6], where spherical Artin groups were said to be "of finite type".) The poset of spherical subsets of I is denoted by $\mathcal{S}(I)$ or simply $\mathcal{S}$. The subposet of non-empty spherical subsets is an abstract simplicial complex which will be denoted $L$, and called the nerve of $M$ (or the nerve of $\left(W,\left\{s_{i}: i \in I\right\}\right)$ ). Thus the vertex set of $L$ is $I$ and a subset $\sigma$ of $I$ spans a simplex of $L$ if and only if $\sigma \in \mathcal{S}$ if and only if $W_{\sigma}$ is finite. (Greek letters such as $\sigma$ or $\tau$ will be used to denote spherical subsets of $I$ when viewed as simplices of $L$.)

Example 1. If $W$ is finite, then $L$ is a simplex of dimension Card $(I)-1$.

Suppose now that $m_{i j}=\infty$ for all $i \neq j$, and let $n=\operatorname{Card}(I)$. In this case $W$ is the free product of $n$ copies of $\mathbb{Z} / 2, A$ is a free group of rank $n$, and $L$ is a 0 -dimensional complex consisting of $n$ points.

A Coxeter matrix is right-angled if each off-diagonal entry is either 2 or $\infty$. The associated Coxeter group and Artin group are also said to be right-angled.

For any subset $J$ of $I$ and $w \in W_{I}$, the following two conditions are equivalent:

(1) $w$ is the (unique) element of shortest word length in $w W_{J} \in$ $W_{I} / W_{J}$ 
(2) for each $j \in J, l\left(w s_{i}\right)=l(w)+1$ (where $l(w)$ denotes the word length of $w$ ).

If $w$ satisfies either condition, then it is called $J$-reduced. Moreover, if $w$ is $J$-reduced and $v \in W_{J}$, then $l(w v)=l(w)+l(v)$ (cf. [2, Ex. 3, p. 37]). For each pair $J, J^{\prime}$ of subsets of $I$ with $J \subseteq J^{\prime}$, let $W_{J^{\prime}}^{J}$ denote the set of $J$-reduced elements of $W_{J^{\prime}}$.

We recall now a formula from $[22,11]$. Given a pair of spherical subsets $\tau, \sigma$ with $\tau \leq \sigma$, define an element $T_{\sigma}^{\tau} \in \mathbb{Z} A_{\sigma}$ by the formula

$$
T_{\sigma}^{\tau}:=\sum_{w \in W_{\sigma}^{\tau}}(-1)^{l(w)} a_{w} .
$$

Lemma 9. For any three spherical subsets, $\tau, \sigma, \rho \in \mathcal{S}$ with $\tau \leq \sigma \leq \rho$, we have

$$
T_{\rho}^{\sigma} \cdot T_{\sigma}^{\tau}=T_{\rho}^{\tau} .
$$

Proof. If $u \in W_{\rho}^{\sigma}$ and $v \in W_{\sigma}^{\tau}$, then it may be shown that $l(u v)=$ $l(u)+l(v)$ (see Ex. 3 on p.37 of [2].) It follows that $a_{u} a_{v}$ equals $a_{u v}$. Since, for each $i \in \tau, l\left(u v s_{i}\right)=l(u)+l\left(v s_{i}\right)=l(u)+l(v)+1=l(u v)+1$, the element $u v$ is $\tau$-reduced. Hence, as $u$ ranges over $W_{\rho}^{\sigma}$ and $v$ ranges over $W_{\sigma}^{\tau}$, uv ranges over $W_{\rho}^{\tau}$. Therefore,

$$
\begin{aligned}
& T_{\rho}^{\sigma} \cdot T_{\sigma}^{\tau}=\left(\sum_{u \in W_{\rho}^{\sigma}}(-1)^{l(u)} a_{u}\right)\left(\sum_{v \in W_{\sigma}^{\tau}}(-1)^{l(v)} a_{v}\right) \\
&=\sum_{w \in W_{\rho}^{\tau}}(-1)^{l(w)} a_{w} \\
&=T_{\rho}^{\tau} \cdot \\
& T_{\rho}^{\sigma} \cdot T_{\sigma}^{\tau}=\left(\sum_{u \in W_{\rho}^{\sigma}}(-1)^{l(u)} a_{u}\right)\left(\sum_{v \in W_{\sigma}^{\tau}}(-1)^{l(v)} a_{v}\right)=\sum_{w \in W_{\rho}^{\tau}}(-1)^{l(w)} a_{w} .
\end{aligned}
$$

\section{Some homological Algebra}

Let $L$ be a finite abstract simplicial complex. Later we shall assume that $L$ is ordered, in the sense that there is a given linear ordering of its vertex set. The face category $\mathcal{F}(L)$ of $L$ is defined to be the category whose objects are the simplices of $L$, with one morphism from $\tau$ to $\sigma$ whenever $\tau$ is a face of $\sigma$. The augmented face category $\mathcal{F}^{+}(L)$ has the same objects and morphisms as $\mathcal{F}(L)$, but also has one extra object (the "-1-simplex", denoted $\emptyset$ ) equipped with one morphism to each object. If $L$ has vertex set $I$, each of $\mathcal{F}(L)$ and $\mathcal{F}^{+}(L)$ is isomorphic 
to a full subcategory of the category of subsets of $I$. A cosheaf on $L$ with values in a category $\mathcal{C}$ is a covariant functor $F$ from $\mathcal{F}^{+}(L)$ to $\mathcal{C}$. In the cases of interest to us, $\mathcal{C}$ will be either abelian groups or Hilbert $G$-modules. A homomorphism of cosheaves on $L$ is given by $\phi: F \rightarrow F^{\prime}$, a natural transformation from $F$ to $F^{\prime}$.

If $\tau \leq \sigma$ are faces of $L$ (possibly including the -1 -simplex), let $\iota_{\sigma}^{\tau}$ denote the morphism in $\mathcal{F}(L)$ from $\tau$ to $\sigma$. The morphisms of $\mathcal{F}(L)$ are generated by those $\iota_{\sigma}^{\tau}$ such that $\tau$ is a codimension one face of $\sigma$, and all relations between the morphisms are consequences of the relations

$$
\iota_{\tau^{\prime}}^{\sigma^{\prime}} \iota_{\sigma}^{\tau^{\prime}}=\iota_{\tau}^{\sigma^{\prime}} \iota_{\sigma}^{\tau}
$$

where $\sigma^{\prime}$ is a codimension two face of $\sigma$, and $\tau, \tau^{\prime}$ are the two codimension one faces of $\sigma$ containing $\sigma^{\prime}$. To define a cosheaf $F$ on $L$ it suffices to specify the objects $F(\sigma)$, and morphisms $f_{\sigma}^{\tau}=F\left(\iota_{\sigma}^{\tau}\right): F(\sigma) \rightarrow F(\sigma)$ for all codimension one pairs, so that relations (4) are satisfied for all codimension two pairs.

Now suppose that $L$ is ordered. Then for any $n \geq 0$ the vertices of an $n$-simplex form an ordered set isomorphic to $0<1<\cdots<n$. For any $0 \leq i \leq n$ and any $n$-simplex $\sigma$, the $i$ th face of $\sigma$ is defined to be the $(n-1)$-simplex spanned by all vertices of $\sigma$ except the $i$ th. If one writes $\partial_{i}=\iota_{\tau \sigma}$, where $\tau$ is the $i$ th face of $\sigma$, then the relations between the morphisms become the famililar "cosimplicial identities" [24] 8.1.

A cosheaf $F$ of abelian groups on an ordered simplicial complex $L$ gives rise to a cochain complex $C^{*}(L ; F)$ defined as follows: $C^{n}=0$ for $n<-1$, and for $n \geq-1$,

$$
C^{n}=\bigoplus_{\tau \in L^{i}} F(\tau)
$$

where the indexing set is the set of $n$-simplices of $L$. Under the natural isomorphism

$$
\operatorname{Hom}\left(C^{n}, C^{n+1}\right) \cong \bigoplus_{\tau \in L^{n}, \sigma \in L^{n+1}} \operatorname{Hom}(F(\tau), F(\sigma)),
$$

the coboundary map $d: C^{n} \rightarrow C^{n+1}$ corresponds to the matrix $\left(d_{\tau \sigma}\right)$, where $d_{\tau \sigma}=0$ unless $\tau$ is a face of $\sigma$, and is equal to $(-1)^{i} F\left(\iota_{\sigma}^{\tau}\right)$ if $\tau$ is the $i$ th face of $\sigma$.

Example 2. For any abelian group $N$, and any simplicial complex $L$, there is a constant cosheaf on $L$ denoted by $\underline{N}$, which sends each object of $\mathcal{F}^{+}(L)$ to $N$ and each morphism to the identity map. The cohomology of the corresponding cochain complex is isomorphic to the reduced cohomology of $L$ with coefficients in $N$ :

$$
H^{*} C^{*}(L ; \underline{N})=\bar{H}^{*}(L ; N) .
$$


Now suppose that $M$ is a Coxeter matrix with nerve $L$ and Artin group $A$, and let $N$ be a $\mathbb{Z} A$-module. Define a cosheaf $\mathcal{N}$ on $L$ by sending each object to $N$, and for each $\tau<\sigma$ sending the morphism $\iota_{\sigma}^{\tau}$ to left multiplication by $T_{\sigma}^{\tau}$. (Lemma 9 implies that this does define a cosheaf.)

One of the key observations of this paper is:

Lemma 10. For any Coxeter matrix, $M$, with $L, A$ and $N$ as above, there is a cosheaf homomorphism $\phi: \underline{N} \rightarrow \mathcal{N}$ defined by

$$
\phi(\sigma): n \mapsto T_{\sigma}^{\emptyset} \cdot n .
$$

Proof. Immediate from Lemma 9.

Corollary 11. Let $N$ be a $\mathbb{Z} A$-module upon which $T_{\sigma}^{\emptyset}$ acts isomorphically for each $\sigma \in L$. Then

$$
H^{*} C^{*}(L ; \mathcal{N}) \cong \bar{H}^{*}(L ; N)
$$

Proof. In this case the map described in Lemma 10 is an isomorphism of cosheaves, and so induces an isomorphism of cochain complexes $C^{*}(L ; \underline{N}) \rightarrow C^{*}(L ; \mathcal{N})$.

\section{The Salvetti Complex}

Let $M$ be a spherical Coxeter matrix on a set $I$ of cardinality $n$. By definition the associated Coxeter group $W$ is finite, and so admits a standard representation as an orthogonal reflection group on $\mathbb{R}^{n}$. In this case, Salvetti $[22,23]$ defined a regular CW-complex $X^{\prime}$ which is equivariantly homotopy equivalent to $Y$, the complexification of $\mathbb{R}^{n}$ minus the hyperplanes fixed by reflections in $W$. Each closed cell of $X^{\prime}$ is naturally identified with a face of a certain convex polytope associated to $W$ (namely the convex hull of a generic $W$-orbit in $\mathbb{R}^{n}$ ). Such a polytope is called a "Coxeter cell". The group $W$ acts freely on $X^{\prime}$. The quotient space, denoted by $X_{I}$ or $X$, is called the Salvetti complex, and has fundamental group $A$.

As mentioned in the introduction, the definition of $X$ was extended to arbitrary Coxeter matrices in [7]. As before, there is a regular $W$ CW-complex $X^{\prime}$ such that each closed cell is a Coxeter cell and such that $X^{\prime}$ is homotopy equivalent to a suitably defined hyperplane complement $Y$. The quotient space $X=X^{\prime} / W$ is the Salvetti complex, and has fundamental group $A$. The space $X$ has one open cell $e_{\sigma}$ for each spherical subset $\sigma$ of $I$. Moreover the closure of $e_{\sigma}$ in $X$ is the subcomplex $X_{\sigma}$ corresponding to the spherical Artin group $A_{\sigma}$. 
Example 3. Let $\mathbb{T}^{n}$ denote the usual cubical structure on the $n$-torus, with one $k$-dimensional cell for each subset of $I=\{1, \ldots, n\}$ of cardinality $k$. In the case when $W=(\mathbb{Z} / 2)^{n}$, then $A=\mathbb{Z}^{n}$, and $X=\mathbb{T}^{n}$.

More generally, if $A$ is right-angled with generating set $I$ of cardinality $n$, then $X$ is a subcomplex of $\mathbb{T}^{n}$, consisting of those cells of $\mathbb{T}^{n}$ that correspond to spherical subsets of $I$.

It is proved in [7] that the poset of cells in $X^{\prime}$ can be identified with $W \times \mathcal{S}$, where the partial order given by incidence of faces is given by $(w, \tau)<(v, \sigma)$ if and only if $\tau<\sigma$ and $v^{-1} w \in W_{\sigma}^{\tau}$. Henceforth we identify a cell with the corresponding element of $W \times \mathcal{S}$. The cell corresponding to $(v, \sigma)$ is a Coxeter cell associated to $W_{\sigma}$; its vertex set is the set $\left\{(v u, \emptyset): u \in W_{\sigma}^{\emptyset}\right\}$ and its dimension is $\operatorname{Card}(\sigma)$.

An edge or 1-cell of $X^{\prime}$ has the form $(w,\{i\})$ for some $w \in W$ and $i \in I$. Orient this edge by declaring $(w, \emptyset)$ to be its initial vertex and $\left(w s_{i}, \emptyset\right)$ to be its terminal vertex. Since the $W$-action preserves the edge orientations, this gives rise to an orientation of the edges of $X=X^{\prime} / W$. Let $\left\{e_{i}: i \in I\right\}$ denote the set of edges of $X$ with their induced orientation. Since $X$ has only one vertex, each $e_{i}$ gives rise to an oriented loop. By definition the generator $a_{i}$ of the Artin group $A=\pi_{1}(X)$ is the homotopy class of the oriented loop around $e_{i}$.

A vertex $x$ of a cell $C$ in $X^{\prime}$ is called a top vertex (resp. bottom vertex) of $C$ if each edge of $C$ that contains $x$ points away from $x$ (resp. towards $x)$. Each cell $(v, \sigma)$ of $X^{\prime}$ has a unique top vertex, $(v, \emptyset)$, and a unique bottom vertex $\left(v u_{\sigma}, \emptyset\right)$, where $u_{\sigma}$ denotes the element of longest length in the finite Coxeter group $W_{\sigma}$.

Let $\widetilde{X}$ denote the universal cover of $X$ (or equivalently of $X^{\prime}$ ). The orientations of the edges of $X^{\prime}$ lift to orientations on the edges of $\widetilde{X}$. Each cell of $\widetilde{X}$ then also has a unique top vertex and a unique bottom vertex. The 1-skeleton of $\widetilde{X}$ is an oriented graph and each edge is labelled by an element of $\left\{a_{i}: i \in I\right\}$ so that if $e$ is an oriented edge labelled by $a_{i}$ whose initial vertex is $x$, then the terminal vertex of $e$ is $x \cdot a_{i}$.

Now fix a lift $\widetilde{x} \in \tilde{X}$ of the vertex $(1, \emptyset)$ of $X^{\prime}$. This gives an identification of the vertex set of $\widetilde{X}$ with $A$ and of the 1 -skeleton of $\widetilde{X}$ with the Cayley graph of the standard presentation of $A$.

For each $\sigma \in \mathcal{S}$, let $\widetilde{e}_{\sigma}$ be the lift of the cell $(1, \sigma)$ whose top vertex is $\widetilde{x}$. Using the $A$-action we get an identification of the poset of cells in $\tilde{X}$ with $A \times \mathcal{S}$. The partial order on $A \times \mathcal{S}$ corresponding to "is a face of" is defined by $(b, \tau)<(a, \sigma)$ if and only if $a=b a_{u}$ for some 
$u \in W_{\sigma}^{\tau}$. Thus for a given cell $(a, \sigma)$ of $\tilde{X}$, its set of faces of type $\tau$ is $\left\{\left(a a_{u}, \tau\right): u \in W_{\sigma}^{\tau}\right\}$.

Once we have fixed an ordering on $I$ there is an induced orientation for each cell of $\widetilde{X}$ : a cell $C$ with top vertex $x$ is oriented so that the edges at $x$ in their natural order give an oriented basis for the tangent space of $C$ at $x$ (recall that $C$ may be viewed as a convex polytope in $\mathbb{R}^{n}$ for some $n$ ). Suppose that $C$ is of type $\sigma, \sigma=\left\{i_{1}<\cdots<i_{k}\right\}$ and that $D$ is a codimension one face of type $\tau=\sigma-\left\{i_{j}\right\}$. Suppose also that $D$ has the same top vertex as $C$. In this case the natural orientation of $D$ and its orientation induced by the orientation on $C$ (by taking an outward pointing normal) differ by the sign $\epsilon(\sigma, \tau)$ defined by $\epsilon(\sigma, \tau)=(-1)^{j-1}$.

If $(v, \sigma)$ is a cell of $X^{\prime}$, then the (right) action of $W_{\sigma}$ on the cell (induced by the action on its vertex set) is such that the action of $u \in W_{\sigma}$ changes the sign of the orientation by $(-1)^{l(u)}$. Hence we have a formula for the boundary maps in the cellular chain complex $C_{*}\left(X^{\prime}\right)$ :

$$
\partial(v, \sigma)=\sum_{\tau} \epsilon(\sigma, \tau) \sum_{u \in W_{\sigma}^{\tau}}(-1)^{l(u)}(v u, \tau),
$$

where the summation is over all codimension one faces $\tau$ of $\sigma$. Similarly, we have a formula for the boundary maps in $C_{*}(\widetilde{X})$ :

$$
\begin{aligned}
\delta\left(a \widetilde{e}_{\sigma}\right)=\delta(a, \sigma) & =\sum_{\tau} \epsilon(\sigma, \tau) \sum_{u \in W_{\sigma}^{\tau}}(-1)^{l(u)}\left(a a_{u}, \tau\right) \\
& =a \sum_{\tau} \widetilde{e}_{\tau} T_{\sigma}^{\tau} \cdot \epsilon(\tau, \sigma) .
\end{aligned}
$$

Remark. Note the similarity with the formula occuring in Lemma 9. An alternative proof of Lemma 9 can be given by considering the oriented incidence relation between cells in $\widetilde{X}$ of types $\tau, \sigma$ and $\rho$, where $\tau \subseteq$ $\sigma \subseteq \rho$.

The cells $\widetilde{e}_{\sigma}$ for $\sigma \in \mathcal{S}$ form a $\mathbb{Z} A$-basis for the cellular chain groups of $\widetilde{X}$. In matrix terms, the $i$ th cellular chain group of $\widetilde{X}$ may be viewed as consisting of row vectors over $\mathbb{Z} A$, with the standard basis elements corresponding to the cells $\widetilde{e}_{\sigma}$, where $\sigma$ ranges over the subsets of $\mathcal{S}$ of size $i$. The $\mathbb{Z} A$-action is the standard left action, and the boundary map in the chain complex is given by right multiplication by a matrix whose entry in the $(\sigma, \tau)$-position is $\epsilon(\tau, \sigma) T_{\sigma}^{\tau}$ if $\tau \subseteq \sigma$ and is zero otherwise.

Now suppose that $N$ is any $\mathbb{Z} A$-module. After identifying $C_{i}(\widetilde{X})$ with a space of row vectors with entries in $\mathbb{Z} A$ as above, it is natural 
to identify $\operatorname{Hom}_{A}\left(C_{i}(\widetilde{X}), N\right)$ with a space of column vectors (of the same length) with entries in $N$, so that the evaluation map corresponds to multiplying a row vector and a column vector to produce a single element of $N$. In these terms, the coboundary map in the cochain complex $\operatorname{Hom}_{A}\left(C_{*}(\widetilde{X}), N\right)$ is described as left multiplication by the same matrix over $\mathbb{Z} A$ as used previously to describe the boundary map in $C_{*}(\widetilde{X})$. This gives the following theorem.

Theorem 12. For any $A$-module $N$, there is an isomorphism between $C^{*}(X ; N)=\operatorname{Hom}_{A}\left(C_{*}(\widetilde{X}), N\right)$ as defined at the beginning of Section 3 and $C^{*-1}(L ; \mathcal{N})$ as defined in Section 6.

In the spherical case, a statement equivalent to the above theorem is contained in $[23,11]$, and it is used to compute some cohomology.

Remark. There is a simple description of the CW-complex $X$. Firstly, for each $\sigma \in \mathcal{S}$ we describe a CW-complex $X_{\sigma}$. Start with a Coxeter cell $C_{\sigma}$ of type $W_{\sigma}$. For each $\tau<\sigma$ identify $C_{\tau}$ with the face of $C_{\sigma}$ of type $\tau$ which has the same top vertex as $C_{\sigma}$. Now for each $\tau<\sigma$ and each $u \in W_{\sigma}^{\tau}$ glue together $C_{\tau}$ and $u C_{\tau}$ via the homeomorphism induced by $u$. The result is $X_{\sigma}$. To construct $X$, start with the disjoint union of the $X_{\sigma}$ for $\sigma \in \mathcal{S}$, and then use the natural maps to identify $X_{\tau}$ with a subcomplex of $X_{\sigma}$ whenever $\tau<\sigma$.

Remark. In section 3 of [6] it is shown how to associate a "simple complex of groups" $\mathcal{A}$ to a Coxeter matrix. The underlying poset is $\mathcal{S}$. To each $\sigma \in \mathcal{S}$ one associates the Artin group $A_{\sigma}$, and for each $\tau<\sigma$, the associated homomorphism $A_{\tau} \rightarrow A_{\sigma}$ is the natural inclusion. There is a natural projection from (the barycentric subdivision of) $X$ to the geometric realization of $\mathcal{S}$ such that the inverse image of the vertex corresponding to $\sigma$ is a copy of $X_{\sigma}$. Since it follows from [12] that $X_{\sigma} \simeq B A_{\sigma}$, it follows that $X$ is an aspherical realization of the complex of groups $\mathcal{A}$ in the sense of [17]. Thus $X$ is homotopy equivalent to the classifying space $B \mathcal{A}$ of $\mathcal{A}$ (which is defined as a homotopy colimit of $B A_{\sigma}$ over the category $\mathcal{S}$ ). The main conjecture of [6] is that $B \mathcal{A}$ (or equivalently $X$ ) is aspherical. This has been proved in many cases: for example, it holds if the simplicial complex $L$ is a flag complex (e.g., if the Coxeter matrix is either spherical or right-angled) and it holds whenever the dimension of $L$ is at most 1 .

\section{Cohomology with Generic CoefFicients}

This section represents a slight digression, although the methods used are similar to those that will be applied to $\ell^{2}$-cohomology in later sections. 
Let $A$ be an Artin group with Artin generators $\left\{a_{i}: i \in I\right\}$, let $G$ be the abelianization of $A$, and let and let $\alpha_{i}$ be the image of $a_{i}$ in $G$. In the case when $m_{i j}$ is even, the abelianization of the Artin relator between $a_{i}$ and $a_{j}$ is trivial, and in the case when $m_{i j}$ is odd the abelianization of the Artin relator is $\alpha_{i}=\alpha_{j}$. Hence one sees that $G$ is free abelian, of rank equal to the number of components of the graph with vertex set $I$ and an edge joining $i$ to $j$ if and only if $m_{i j}$ is odd. In particular, $G$ is never trivial. Let $m$ denote the rank of $G$, and (after changing the ordering on $I$ ), suppose that $\alpha_{1}, \ldots, \alpha_{m}$ freely generate $G$.

Now suppose that $k$ is a field of characteristic zero, and that $N$ is a $k A$-module whose underlying $k$-vector space is 1 -dimensional. The action of $A$ on $N$ factors through $G$, a free abelian group of rank $m$, and so $N$ is classified up to isomorphism by an $m$-tuple

$$
\underline{\lambda}=\left(\lambda_{1}, \ldots, \lambda_{m}\right) \in\left(k^{*}\right)^{m},
$$

where $k^{*}=k-\{0\}$ and for $1 \leq i \leq m$, the generator $\alpha_{i}$ acts on $N$ as multiplication by $\lambda_{i}$. As an illustration of the methods we shall apply to $\ell^{2}$-cohomology, we shall compute the cohomology of the Salvetti complex $X$ with local coefficients $N$ for "generic $N$ ", i.e., for $N$ corresponding to elements of a dense Zariski open subset of $\left(k^{*}\right)^{m}$.

Theorem 13. For a generic 1-dimensional $k A$-module $N$, there is an isomorphism

$$
\bar{H}^{*-1}(L ; k) \cong H^{*}(X ; N)
$$

Proof. Each side of the supposed isomorphism is isomorphic to the cohomology of $L$ with coefficients in a certain cosheaf:

$$
\bar{H}^{*}(L ; k) \cong \bar{H}^{*}(L ; N) \cong H^{*} C^{*}(L ; \underline{N})
$$

and by Theorem 12 ,

$$
C^{*}(X ; N) \cong C^{*-1}(L ; \mathcal{N}) .
$$

It therefore suffices to show that for generic $N$, the cosheaf homomorphism $\phi: \underline{N} \rightarrow \mathcal{N}$ described in Lemma 10 is an isomorphism. Equivalently, it suffices to show that for each simplex $\sigma$ of $L$, multiplication by $T_{\sigma}^{\emptyset}$ is an isomorphism for generic $N$.

For $\underline{\lambda}=\left(\lambda_{1}, \ldots, \lambda_{m}\right) \in\left(k^{*}\right)^{m}$, let $\psi_{\underline{\lambda}}^{\prime}: \mathbb{Z} G \rightarrow k$ be defined by $\psi_{\lambda}^{\prime}\left(a_{i}\right)=\lambda_{i}$, and let $\psi_{\underline{\lambda}}: \mathbb{Z} A \rightarrow k$ be the composite of the projection $\mathbb{Z} A \rightarrow \mathbb{Z} G$ and $\psi^{\prime}$. For each $\sigma \in L$, the set of $\underline{\lambda}$ such that $\psi_{\underline{\lambda}}\left(T_{\sigma}^{\emptyset}\right)=0$ is a closed subset of $\left(k^{*}\right)^{m}$. The union over all $\sigma$ of these closed sets is not the whole of $\left(k^{*}\right)^{m}$, since in the case when $\underline{\lambda}=(-1,-1, \cdots,-1)$, $\psi_{\underline{\lambda}}\left(T_{\sigma}^{\emptyset}\right)=\operatorname{Card}\left(W_{\sigma}\right)$. Since $\left(k^{*}\right)^{m}$ is connected, it follows that the set of $\underline{\lambda}$ for which each $\psi_{\underline{\lambda}}\left(T_{\sigma}^{\emptyset}\right) \neq 0$ is open and dense in $\left(k^{*}\right)^{m}$ as required. 
Remark. The theorem does not hold for arbitrary 1-dimensional coefficients, as can be seen by comparing the case of "generic" 1-dimensional coefficients $N$ with the trivial 1-dimensional module $k$. As a first example, consider $H^{1}$. For any $A, H^{1}(A ; k)$ is naturally isomorphic to $\operatorname{Hom}(A, k)$, which has $k$-dimension equal to the rank of the abelianization of $A$. In particular it is non-zero for every Artin group, whereas for $A$ of spherical type and generic $N$, we have seen that $H^{*}(A ; N)=\{0\}$. (Note that in the case when $A$ is of spherical type, $H^{*}(A ; k)$ is computed in [4].)

As a further example, consider the case when $A$ is right-angled. In this case it may be shown that $H^{*}(A ; k)$ is isomorphic to the "exterior face ring of $L$ ", defined by taking the exterior algebra over $k$ with generators $\left\{x_{i}: i \in I\right\}$ with each $x_{i}$ in degree 1 , and adding in the relation $x_{i_{1}} \cdots x_{i_{m}}=0$ whenever $i_{1}, \ldots, i_{m}$ do not form the vertex set of a simplex of $L[18,13]$. In particular, the rank of $H^{i}(X ; k)$ is equal to the number of $i$-simplices in $L$ for each $i>0$.

\section{Reduced $\ell^{2}$-COHOMOLOGY}

Each Artin group of spherical type has a non-trivial centre. If $A$ is a spherical Artin group and $w$ is the longest element of the corresponding Coxeter group, then the element $\left(a_{w}\right)^{2}$ is in the centre of $A$ (c.f. [12]). Note that the expression for this element contains only positive powers of the Artin generators, and so in particular its image under the standard homomorphism $A \rightarrow \mathbb{Z}$ is non-zero.

Lemma 14. Suppose that $A$ is an Artin group of spherical type, and that $A^{\prime}$ is a normal subgroup of $A$ contained in the kernel of the standard homomorphism. Let $X^{\prime} \rightarrow X$ be the corresponding covering space of $X$ and let $G=A / A^{\prime}$. For each $i$, the Hilbert $G$-module $\mathcal{H}^{i}\left(X^{\prime}\right)$ is the zero module.

Proof. In view of the remarks preceding the statement of Lemma 14, this is a special case of Corollary 7 .

Proposition 15. Let $H \leq G$, and let $\xi \in \mathbb{Z} H$. Then left multiplication by $\xi$ induces a weak isomorphism of $\ell^{2}(H)$ if and only if it induces a weak isomorphism of $\ell^{2}(G)$.

Proof. Consider $\mathcal{H}$, defined to be the set of all functions on $G$ that are square-summable on each right coset $H g$ of $H$. As a left $\mathbb{Z} H$ module, $\mathcal{H}$ is isomorphic to a direct product of copies of $\ell^{2}(H)$ indexed by the coset space $H \backslash G$. One has that $\ell^{2}(H) \leq \ell^{2}(G) \leq \mathcal{H}$, and so multiplication by $\xi$ is injective as a self-map of $\ell^{2}(H)$ if and only if it 
is injective as a self-map of $\ell^{2}(G)$. The corresponding assertion with "weak isomorphism" in place of "injective" follows from Lemma 4.

Lemma 16. Suppose that $A=A_{\sigma}$ is an Artin group of spherical type, and that $A^{\prime}$ is a normal subgroup of $A$ contained in the kernel of the standard homomorphism $A \rightarrow \mathbb{Z}$. Let $G=G_{\sigma}=A / A^{\prime}$, and let $\phi_{\sigma}$ : $\ell^{2}(G) \rightarrow \ell^{2}(G)$ denote left multiplication by the element $T_{\sigma}^{\emptyset} \in \mathbb{Z} A_{\sigma}$. Then $\phi_{\sigma}$ is a weak isomorphism.

Proof. Let $\pi$ denote the quotient map from $A_{\sigma}$ to $G_{\sigma}$. For any $\tau<\sigma$, let $i_{\tau}$ denote the inclusion of $A_{\tau}$ in $A_{\sigma}$, and define a subgroup $G_{\tau} \leq G_{\sigma}$ by $G_{\tau}=\pi \circ i_{\tau}\left(A_{\tau}\right)$. The composite of $i_{\tau}$ and the standard homomorphism for $A_{\sigma}$ is equal to the standard homomorphism for $A_{\tau}$, and so the pair $\left(A_{\tau}, G_{\tau}\right)$ satisfy the hypotheses of the Lemma.

The lemma is trivially satisfied if $\operatorname{Card}(\sigma)=0$, since $T_{\emptyset}^{\emptyset}=1$. Hence by induction on $\operatorname{Card}(\sigma)$, we may assume that for any $\tau<\sigma$, the map $\phi_{\tau}: \ell^{2}\left(G_{\tau}\right) \rightarrow \ell^{2}\left(G_{\tau}\right)$ is a weak isomorphism. Here $\phi_{\tau}$ is defined to be left multiplication by the element $T_{\tau}^{\emptyset} \in \mathbb{Z} A_{\tau}$. By Proposition 15 , we may assume that $\phi_{\tau}: \ell^{2}\left(G_{\sigma}\right) \rightarrow \ell^{2}\left(G_{\sigma}\right)$ is also a weak isomorphism.

Now let $L$ be the simplex with vertex set $\sigma$, where $n=\operatorname{Card}(\sigma)$. Let $N$ denote $\ell^{2}\left(G_{\sigma}\right)=\ell^{2}(G)$, and consider the cosheaves of Hilbert $G$-modules on $L$ that were denoted by $\underline{N}$ and $\mathcal{N}$ in Section 6 . Note that in this case, $C^{*}(L ; \underline{N})$ and $C^{*}(L ; \overline{\mathcal{N}})$ are cochain complexes of Hilbert $G$-modules. Since the reduced cohomology of $L$ is trivial, the cochain complex $C^{*}(L ; \underline{N})$ is exact. By Theorem 12 the cochain complex $C^{*}(L ; \mathcal{N})$ (with a shift of degree) may be used to compute the cohomology of $A$ with coefficients in $\ell^{2}(G)$, and so by Lemma 14, it follows that $C^{*}(L ; \mathcal{N})$ is weakly exact.

Now consider the cosheaf homomorphism $\phi: \underline{N} \rightarrow \mathcal{N}$ defined in Lemma 10, and the induced map

$$
\phi^{*}: C^{*}(L ; \underline{N}) \rightarrow C^{*}(L ; \mathcal{N}) .
$$

In degree $i$, each of $C^{i}(L ; \underline{N})$ and $C^{i}(L ; \mathcal{N})$ is isomorphic to a direct sum of copies of $\ell^{2}(G)$ indexed by the subsets $\tau \leq \sigma$ with $\operatorname{Card}(\tau)=i$. With respect to these bases, the map $\phi^{i}$ is given by a diagonal matrix, whose $(\tau, \tau)$-entry is the map $\phi_{\tau}$. By induction, this map is a weak isomorphism in each degree except possibly $n$, the top degree. Consider the following commutative diagram, consisting of the right-hand ends of the two cochain complexes.

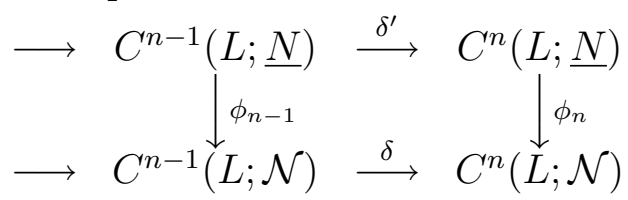


This can be rewritten as follows, where $\tau$ runs over the subsets of $\sigma$ of size $n-1$.

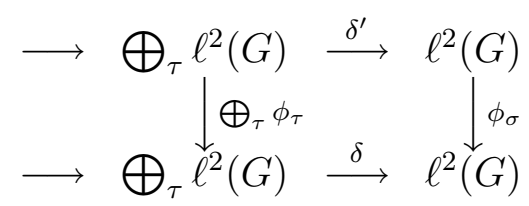

The coboundary map $\delta^{\prime}$ is surjective, the coboundary map $\delta$ is weakly surjective, and by induction the left-hand vertical map is a weak isomorphism. It follows that the right-hand vertical map, $\phi_{\sigma}: \ell^{2}(G) \rightarrow$ $\ell^{2}(G)$ is weakly surjective. The claim now follows from Lemma 4 .

Corollary 17. Let $A$ be any Artin group, let $A^{\prime}$ be a normal subgroup of $A$ contained in the kernel of the standard homomorphism $A \rightarrow \mathbb{Z}$, let $G=A / A^{\prime}$, and let $N=\ell^{2}(G)$. Then the map $\phi: \underline{N} \rightarrow \mathcal{N}$ defined in Lemma 10 is a weak isomorphism of cosheaves of Hilbert $G$-modules on the simplicial complex $L$.

Proof. On each object $\sigma \in \mathcal{S}$, the map $\phi(\sigma): \underline{N}(\sigma) \rightarrow \mathcal{N}(\sigma)$ is equal to left multiplication by $T_{\sigma}^{\emptyset}$. By Lemma 16 and Proposition 15, each of these maps is a weak isomorphism.

The next Corollary is immediate from the above.

Corollary 18. With notation as in Corollary 17, the induced map of cochain complexes

$$
\phi^{*}: C^{*}(L ; \underline{N}) \rightarrow C^{*}(L ; \mathcal{N})
$$

is a weak isomorphism.

The proofs of Theorems 1 and 3 are now completed by combining the description of $\left.\mathcal{H}^{(} X^{\prime}\right)$ as the reduced cohomology of $C^{*}(L ; \mathcal{N})$, given in Theorem 12, with Corollary 18 and Lemma 5.

\section{Closing Remarks}

Although we have chosen to work with real $\ell^{2}$-cohomology throughout, everything that we have done works equally well for complex $\ell^{2}$ cohomology, defined in terms of the complex Hilbert space $\ell_{\mathbb{C}}^{2}(G)$ of functions from $G$ to the complex numbers for which the square of the modulus is summable.

Two other methods for proving Theorems 1 and 3 have been suggested to the authors. One of these, which was suggested by Tadeusz Januszkiewicz, gives some information concerning the $\ell^{2}$-cohomology of arbitrary hyperplane arrangements and will be described in another publication. The other method, suggested to us by Wolfgang Lück and Thomas Schick, involves a spectral sequence argument. The advantage 
of this method seems to be that it requires fewer explicit calculations with the Salvetti complex. However, it appears to give a weaker result than the method used above. To simplify the notation, we shall discuss this proof for Theorem 1 but not for Theorem 3 .

Recall from Section 7 that for a general Artin group A, the Salvetti complex $X$ may be expressed as a union of subcomplexes $X_{\sigma}$, where $\sigma$ ranges over the spherical subsets of the Artin generating set, and $X_{\sigma}$ is a copy of the Salvetti complex for $A_{\sigma}$. Let $Y_{\sigma}$ be the subcomplex of $\widetilde{X}$, the universal cover of $X$, consisting of lifts of cells of $X_{\sigma}$. Each $Y_{\sigma}$ is a free $A$-CW-complex. By Corollary 8 and Proposition 15, the reduced $\ell^{2}$-cohomology of $Y_{\sigma}$ vanishes for $\sigma \neq \emptyset$. In contrast, $Y_{\emptyset}$ is just the 0 -skeleton of $\widetilde{X}$, which consists of a single free $G$-orbit of cells. Hence the reduced $\ell^{2}$-cohomology of $Y_{\emptyset}$ is a copy of $\ell^{2}(G)$ in degree zero.

One may construct a "Mayer-Vietoris double chain complex" $C_{*, *}$ for $\widetilde{X}$ expressed as the union of the $Y_{\sigma}$. Let $\mathcal{L}$ denote the set of maximal simplices in $L$, define $Y(\emptyset)=\widetilde{X}$, and for $\emptyset \neq S \subseteq \mathcal{L}$, define $Y(S)=$ $\bigcap_{\sigma \in S} Y_{\sigma}$. Define $C_{*, 0}$ to be the cellular chain complex for $\tilde{X}$, and for $j>0$ define $C_{*, j}$ to be the direct sum over the subsets $S \subseteq \mathcal{L}$ of size $j$ of the cellular chain complex for $Y(S)$. The boundary maps in $C_{*, *}$ of degree $(-1,0)$ are the boundary maps in the chain complexes $C_{*}(Y(S))$, and the boundary maps of degree $(0,-1)$ are given by the matrices whose $(S, T)$-entry is $\epsilon(S, T)$ times the map induced by the inclusion of $Y(S)$ in $Y(T)$, where $\epsilon(S, T)=(-1)^{i}$ if $T$ is obtained from $S$ by omitting the $i$ th element of $S$ (for some fixed ordering of $\mathcal{L}$ ). By construction, this double complex has trivial homology, since the boundary map of degree $(0,-1)$ is exact. Since each $C_{i, j}$ is free, it follows that for each $i$, the chain complex $C_{i, *}$ is split exact.

Now suppose that $N$ is a $\mathbb{Z} A$-module such that for each $\sigma \in L$, $H^{*}\left(A_{\sigma} ; N\right)=\{0\}$. Define a double cochain complex by

$$
E_{0}^{*, *}=\operatorname{Hom}_{A}\left(C_{*, *}, N\right),
$$

and let $E_{*}^{*, *}$ denote the spectral sequence in which the differential $d_{0}$ is induced by the boundary map of degree $(-1,0)$ on $C_{*, *}$. The boundary map on $E_{0}^{*, *}$ of degree $(0,1)$ is exact, and so the homology of the total complex for $E_{0}^{*, *}$ is zero. It follows that the $E_{\infty}$-page of the spectral sequence is identically zero. The $E_{1}$-page has $E_{1}^{i, 0} \cong H^{i}(A ; N), E_{1}^{i, j}=$ $\{0\}$ if both $i>0$ and $j>0$, and $E_{1}^{0, j}$ is isomorphic to a direct sum of copies of $N$, indexed by those $j$-element subsets of $\mathcal{L}$ such that the intersection of the corresponding simplices of $L$ is empty.

The structure of the $E_{1}$-page implies that $E_{2}^{i, j}=E_{1}^{i, j}$ for $i>0$, and it may be shown that $E_{2}^{0, j} \cong \bar{H}^{j}(L ; N)$. To see this, note that the 
cochain complex $E_{1}^{0, *}$ embeds as a subcomplex in an exact complex $C^{*}$, where $C^{j}$ is isomorphic to a direct sum of copies of $N$ indexed by all $j$-element subsets of $\mathcal{L}$, and the quotient complex $C^{*} / E_{1}^{0, *}$ is isomorphic to the augmented cochain complex for the nerve of the covering of $L$ by the elements of $\mathcal{L}$, shifted in degree by 1 . The long exact sequence in cohomology coming from this short exact sequence of cochain complexes gives the claimed isomorphism.

Since the $E_{\infty}$-page is identically zero, and the only non-zero groups on the $E_{2}$-page are those $E_{2}^{i, j}$ for which either $i=0$ or $j=0$, it follows that the differential $d_{r}$ must be an isomorphism from $E_{r}^{0, r-1}=E_{2}^{0, r-1}$ to $E_{r}^{r, 0}=E_{1}^{r, 0}$. Thus we obtain

Theorem 19. For any $\mathbb{Z} A$-module $N$ such that $H^{*}\left(A_{\sigma} ; N\right)=\{0\}$ for all non-trivial spherical subgroups $A_{\sigma} \leq A$, there is an isomorphism

$$
H^{*}(X ; N) \cong \bar{H}^{*-1}(L ; N) .
$$

One corollary of this is a version of Theorem 13

Corollary 20. Let $k$ be a field of characteristic zero, and let $N$ be a 1-dimensional $k A$-module, such that for each non-trivial spherical subgroup $A_{\sigma}$, the centre of $A_{\sigma}$ acts non-trivially on $N$. Then $H^{*}(X ; N) \cong$ $\bar{H}^{*-1}(L ; N)$.

Proof. By Proposition 6, the action of the centre $Z$ of $A_{\sigma}$ on $H^{*}\left(A_{\sigma}: N\right)$ is trivial. But as a $k Z$-module $H^{i}\left(A_{\sigma} ; N\right)$ is isomorphic to a submodule of a direct sum of copies of $N$. The only possibility is that $H^{*}\left(A_{\sigma} ; N\right)=$ $\{0\}$. Hence Theorem 19 may be applied.

Unfortunately, Theorem 19 does not apply directly to the case when $N=\ell^{2}(A)$, since we have only that the reduced $\ell^{2}$-cohomology groups $\mathcal{H}^{*}\left(A_{\sigma}\right)$ are equal to $\{0\}$. One way around this difficulty is to use W. Lück's equivalence of categories (for any discrete group $G$ ) between the category of Hilbert $G$-modules and the category of projective modules for the group von Neumann algebra $\mathcal{N}(G)$, as explained in chapter 6 of [20]. The von Neumann dimension is defined for projective $\mathcal{N}(G)$-modules in such a way that it is preserved by the above equivalence of categories. Furthermore, von Neumann dimension extends to a dimension function on all (finitely generated) $\mathcal{N}(G)$-modules so that it is additive on extensions, and such that $\operatorname{dim}_{G}(M)=0$ if and only if $\operatorname{Hom}_{G}(M, \mathcal{N}(G))=\{0\}$. For any CW-complex $Y$ with fundamental group $G$, the von Neumann dimension of the ordinary homology group $H_{i}(Y ; \mathcal{N}(G))$ is equal to the von Neumann dimension of $\mathcal{H}^{i}(Y)$. Hence the following statement is a weak version of Theorem 1. 
Theorem 21. For any Artin group $A$ and any $i$, the von Neumann dimensions of $H_{i}(X ; \mathcal{N}(A))$ and $\bar{H}_{i-1}(L ; \mathcal{N}(A)) \cong \mathcal{N}(A) \otimes \bar{H}_{i-1}(L)$ are equal.

Proof. Use the homology version of the Mayer-Vietoris spectral sequence described above. In more detail, define a double chain complex

$$
E_{*, *}^{0}=\mathcal{N}(A) \otimes_{\mathbb{Z} A} C_{*, *} .
$$

The corresponding spectral sequence has the following properties:

(1) $E_{*, *}^{\infty}=\{0\}$;

(2) $E_{0, j}^{2} \cong \bar{H}_{j}(L ; N)$;

(3) $E_{i, 0}^{1} \cong H_{i}(A ; \mathcal{N}(A))$;

(4) For $i, j>0, \operatorname{dim}_{G}\left(E_{i, j}^{1}\right)=0$.

(The fourth of these properties follows from Corollary 7.) It may be shown that for $1<s \leq r$,

$$
\operatorname{dim}_{G}\left(E_{0, r-1}^{s}\right)=\operatorname{dim}_{G}\left(E_{0, r-1}^{2}\right) \quad \text { and } \quad \operatorname{dim}_{G}\left(E_{r, 0}^{s}\right)=\operatorname{dim}_{G}\left(E_{r, 0}^{1}\right) .
$$

Since the $E^{\infty}$-page is identically zero, the map $d^{r}: E_{r, 0}^{r} \rightarrow E_{0, r-1}^{r}$ is an isomorphism, and hence $\operatorname{dim}_{G}\left(E_{r, 0}^{r}\right)=\operatorname{dim}_{G}\left(E_{0, r-1}^{r}\right)$.

\section{REFERENCES}

[1] M. Bestvina and N. Brady, Morse theory and finiteness properties of groups, Invent. Math. 129 (1997), 445-470.

[2] N. Bourbaki, Groupes et Algèbres de Lie, Chapitres 4-6, Masson, Paris, 1981.

[3] M. Bridson and A. Haefliger, Metric Spaces of Non-positive Curvature, Springer-Verlag, Berlin and New York, 1999.

[4] E. Brieskorn, Sur les groupes de tresses, Sém. Bourbaki (1971/72), Springer Lec. Notes in Math. 317, Springer-Verlag, Berlin and New York, 1973, pp. 21-44.

[5] K. S. Brown, Cohomology of Groups, Springer-Verlag, Berlin and New York, 1982.

[6] R. Charney and M.W. Davis, The $K(\pi, 1)$-problem for hyperplane complements associated to infinite reflection groups, J. Amer. Math. Soc. 8 (1995), 597-627.

[7] R. Charney and M.W. Davis, Finite $K(\pi, 1)$ s for Artin groups, Prospects in Topology (ed. F. Quinn), Annals of Math. Studies 138, Princeton Univ. Press, Princeton, 2000, pp. 110-124.

[8] J. Cheeger and M. Gromov, $L_{2}$-cohomology and group cohomology, Topology 25 (1986), 189-215.

[9] M.W. Davis, Nonpositive curvature and reflection groups, Handbook of Geometric Topology (eds. R. Daverman and R. Sher), Elsevier, Amsterdam, 2001.

[10] M.W. Davis and B. Okun, Vanishing theorems and conjectures for the $\ell^{2}$ homology of right-angled Coxeter groups, Geometry and Topology 5 (2001), $7-74$.

[11] C. De Concini and M. Salvetti, Cohomology of Artin groups, Math. Res. Lett. 3 (1996), 293-297. 
[12] P. Deligne, Les immeubles des groupes de tresses généralisés, Invent. Math. 17 (1972), 273-302.

[13] W. Dicks and I. J. Leary, Presentations for subgroups of Artin groups, Proc. Amer. Math. Soc. 127 343-348 (1999).

[14] B. Eckmann, Introduction to $\ell_{2}$-methods in topology: reduced $\ell_{2}$-homology, harmonic chains, $\ell_{2}$-Betti numbers, Israel J. Math. 117 (2000), 183-219

[15] M. Farber, Von Neumann Betti numbers and Novikov type inequalities, Proc. Amer. Math. Soc. 128 (2000), 2819-2827.

[16] M. Gromov, Asymptotic invariants of infinite groups, Geometric Group Theory 2 (eds. G. Niblo and M. Roller), London Math. Soc. Lecture Notes 182, Cambridge Univ. Press, Cambridge, 1993.

[17] A. Haefliger, Extensions of complexes of groups, Ann. Inst. Fourier (Grenoble) 42 (1992), 275-311.

[18] K. H. Kim and F. W. Roush, Homology of certain algebras defined by graphs, J. Pure Appl. Algebra 17, 179-186 (1980).

[19] W. Lück, $L^{2}$-invariants of regular coverings of compact manifolds and $C W$ complexes, Handbook of Geometric Topology (eds. R. Daverman and R. Sher), Elsevier, Amsterdam, 2001.

[20] W. Lück, $L^{2}$-invariants and $K$-theory, Springer-Verlag, Berlin and New York, 2002 .

[21] D. Quillen, Higher algebraic K-theory: I, Battelle Institute Conf., 1972, Springer Lec. Notes in Math. 341, Springer-Verlag, Berlin and New York, 1973, pp. 77-139.

[22] M. Salvetti, Topology of the complement of real hyperplanes in $\mathbb{C}^{n}$, Invent. Math. 88 (1987), 603-618.

[23] M. Salvetti, The homotopy type of Artin groups, Math. Res. Lett. 1 (1994), $565-577$.

[24] C. A. Weibel, An introduction to homological algebra, Cambridge Studies in Advanced Mathematics 38, Cambridge Univ. Press, Cambridge, 1994.

Department of Mathematics, The Ohio State University, $231 \mathrm{~W}$. 18th Avenue, Columbus, Ohio 43210, United States

E-mail address: mdavis@math.ohio-state.edu

Faculty of Mathematical Studies, University of Southampton, Southampton, SO17 1BJ, United Kingdom

E-mail address: I.J.Leary@maths.soton.ac.uk 